\title{
Fellow Column: A Neonate with Fetal Brain Disruption Sequence
}

Andrea Ho, MD, Robin Clark, MD, Gilbert Martin, MD

\section{Introduction:}

Fetal brain disruption sequence (FBDS) is a condition with many causes that is characterized by severe microcephaly of prenatal onset, overlapping sutures, scalp rugae, neurological impairment, normal hair pattern, and occipital bone prominence. FBDS is postulated to result from a disruption in brain development during the second or third trimester, which leads to decreased intracranial hydrostatic pressure with the consequent collapse of the fetal skull. Overlapping sutures, occipital bone prominence, and scalp rugae follow as a result of this collapse. Preservation of hair formation is explained by otherwise normal development during the first 18 weeks of gestation, the period when hair follicles develop.

\section{Case History:}

A two-day-old male was transferred to Loma Linda University Children's Hospital for cyanosis associated with feeding, desaturations, and hypothermia. The mother was a 24-year-old G2P2 female. The mother used tobacco before she came to the knowledge of her pregnancy and did not receive prenatal care. She denied the use of illicit drugs. Based on the last menstrual period, the neonate was 41 weeks 6 days gestation at birth. The mother reported an untreated urinary tract infection and pubic folliculitis at 30-33 weeks of gestation. Parents also stated that they were exposed to mold during the entire pregnancy and that the family moved at 39 weeks of gestation. He was born via spontaneous vaginal delivery at home, where the parents noted that his head was small and misshapen. Paramedics arrived shortly after delivery and transported the neonate to an outside hospital. The parents were told that he had anencephaly, and they brought him home for hospice care. On day two of life, he was at the pediatrician's office breastfeeding when he choked and became cyanotic and limp. He was taken to an outside hospital, where he was hypothermic to $94.3 \mathrm{~F}$, had periodic breathing, desaturated to $84 \%$, and required nasal cannula 1 LPM. He was treated with ampicillin and gentamicin. Chest $x$-ray showed ground-glass opacities. A brain MRI showed diffuse symmetric microcephaly with abnormal sulcation suggestive of a simplified gyral pattern, a $7 \mathrm{~mm}$ subdural hematoma, a thin corpus callosum, mild symmetric dilation of the occipital horns, a diminutive appearance of the optic nerves, and fluid in the mastoid air cells. The neonate was then transferred to our facility for genetics, and pediatric neurology consults as well as for further management of respiratory failure.

\section{"Less than twenty-four hours after} arrival to the pediatrics unit, the neonate had bradycardic events with heart rate in the 80 's and desaturations to $60 \%$ despite tactile stimulation and nasal cannula 1 LPM. A cardiopulmonary arrest was suspected."
Less than twenty-four hours after arrival to the pediatrics unit, the neonate had bradycardic events with heart rate in the 80's and desaturations to $60 \%$ despite tactile stimulation and nasal cannula 1 LPM. A cardiopulmonary arrest was suspected. A code was called, and ROSC was achieved after five cycles of chest compressions with bag-mask ventilation. The neonate was then transferred to the NICU.

The mother of this neonate was 24-years-old at the time of his birth. She is healthy, though she reports having a "small head." She is of Russian, French, German, and Italian descent. The father is 37-years-old. He has numbness of the hands and feet as well as constant migraines, and he has been referred to a neurologist. He is of Spanish and possibly French-Canadian ancestry. The parents denied consanguinity and previous miscarriages. Their head circumferences are unavailable. The neonate has a 3-year-old full sister who is healthy.

The neonate had the following additional physical anomalies: a prominent occipital ridge that extended in the transverse plane, overriding occipital sutures, deep scalp folds, small for gestational age, narrow and sloped forehead, downward slanting palpebral fissures, high and broad nasal bridge, low set and posteriorly rotated ears, micrognathia, dry skin, high-arched and narrow palate, sacral indentation, sustained arm extension when eliciting Moro reflex, absent Babinski reflexes, absent rooting response, and optic nerve hypoplasia of both eyes. The infant's head circumference at two days of life was $26 \mathrm{~cm}$, which represents a $Z$ score (standard deviation) of -7.34 for infants of this gestational age. The first recorded weight available, on day 2 of life, was $2.885 \mathrm{~kg}$, which correlates with a Z-score of -2.49. Due to transient hypertonic episodes followed by periods of low tone, a video EEG was performed, which showed a burst-suppression pattern with gen-

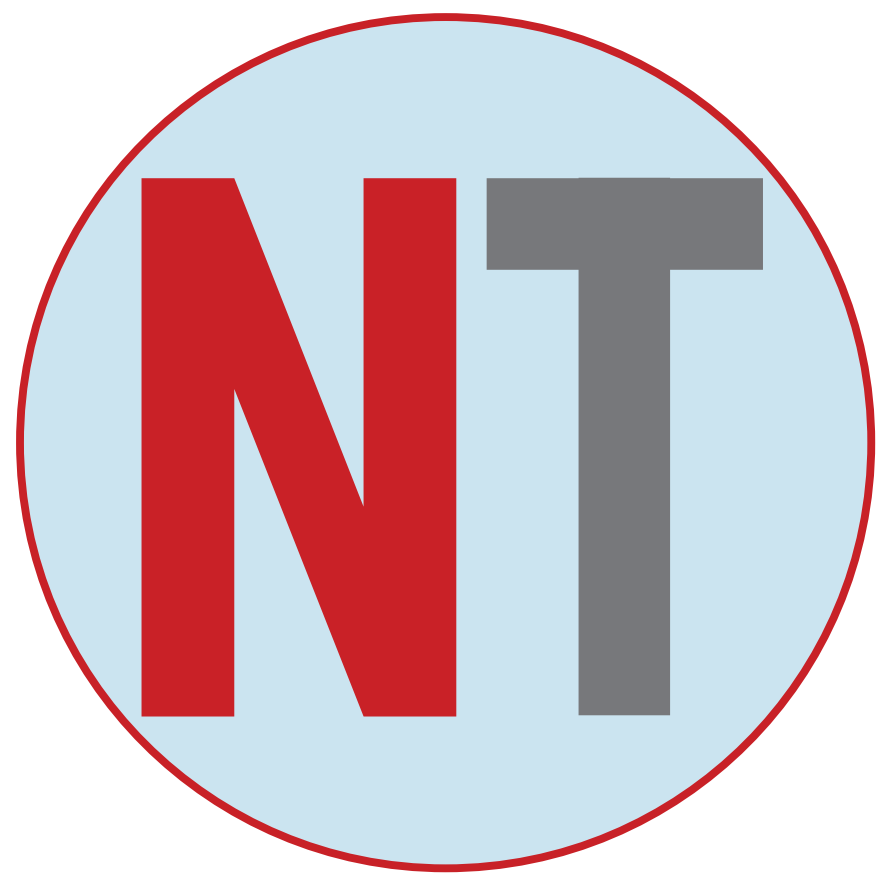




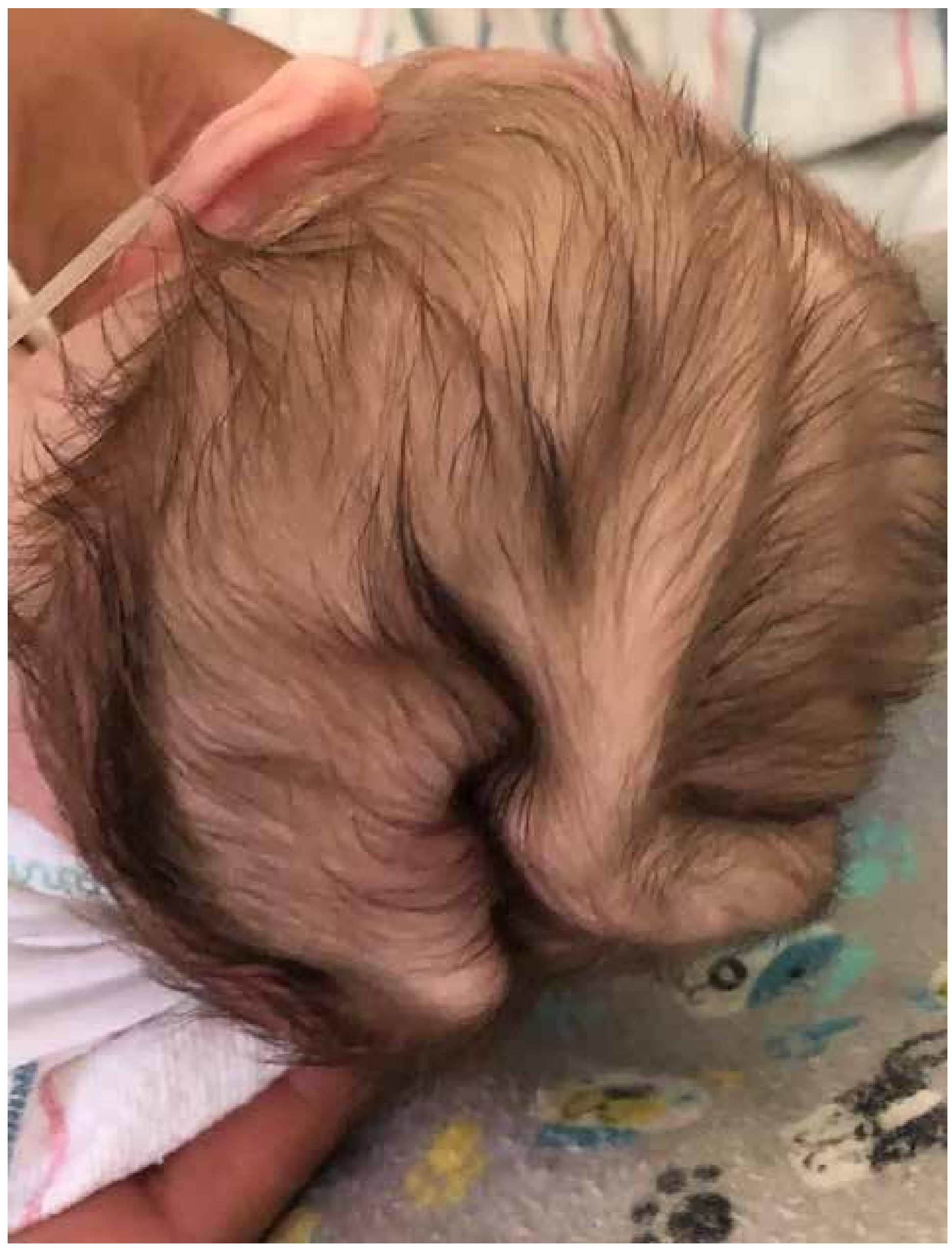


eralized myotonic seizures and generalized tonic seizures consistent with Ohtahara syndrome. Despite the resolution of the subdural hematoma on brain MRI, the neonate's seizures persisted. He was treated with levetiracetam for an indefinite duration as well as phenobarbital. Due to poor feeding, the neonate later required a gastrostomy tube. Due to optic nerve hypoplasia, an evaluation for panhypopituitarism was performed, which showed no abnormalities. An echocardiogram revealed no congenital heart disease.

Laboratory studies were not revealing. The newborn screen was normal. Laboratory tests for congenital infections were negative. Zika virus PCR was negative. IgM and IgG antibodies for CMV and toxoplasma were negative. The chromosomal microarray was normal. Microcephaly Next Generation Sequencing (NGS) panel (Fulgent Diagnostics, 76 genes tested) and Lissencephaly NGS panel (Fulgent Diagnostics, 15 genes tested) also revealed no abnormalities. Plasma amino acids, ammonia, urine organic acid, and acylcarnitine profile were within normal ranges.

\section{"FBDS was first recognized in 1984 in three infants who had a pattern of microcephaly, occipital bone prominence, overlying sutures, and scalp rugae. (7)"}

\section{Discussion:}

FBDS was first recognized in 1984 in three infants who had a pattern of microcephaly, occipital bone prominence, overlying sutures, and scalp rugae. (7) A 2001 review of twenty cases of FBDS identified the most common features of this sequence as normal scalp hair pattern, scalp rugae, overlapped sutures, and occipital bone prominence; microcephaly was a cardinal feature, with an average standard deviation of occipitofrontal circumference of -5.8. (1) Autopsy and CT head findings in these subjects included the destruction of cerebral hemispheres, hydranencephaly, a deficit in cortical tissues, ventriculomegaly, small cerebellum, intracranial calcification, intracranial hemorrhage, porencephaly, colpocephaly, small gyri, and lissencephaly. Proposed mechanisms of FBDS include viral infections, disruptions of vascular supply to the fetal brain, and genetic disorders. When viral infections are implicated, the most common viruses are CMV and Zika virus. Vascular disruption events that cause FBDS include prenatal cocaine exposure, trauma, death of a co-twin, and DIC. (1) Autosomal recessive genetic disorders can cause fetal brain disruption-like phenomenon, such as ALG11-congenital disorder of glycosylation and deletion of $16 \mathrm{p} 13.11$, which can unmask a pathogen variant in the NDE1 gene on the intact homolog, creating a biallelic loss of function for this gene. $(5,6)$

This infant's numerous scalp folds suggested cutis verticis gyrate (CVG) to some examiners. CVG presents as symmetric, redundant scalp folds that exhibit deep furrows and convolutions mimicking that of cerebral gyri. CVG presents in adults or adolescents and is rare in infants and children. It is focal with tightly arranged and fixed folds. It can be seen with psychiatric disorders, seizures, and intellectual disability, but it is not associated with congenital microcephaly. This patient has scalp skin folds that are not characteristic of CVG: they are diffuse, involving the entire scalp. They are loose and are not fixed firmly in place.
This neonate's prominently rugated scalp, overlapping sutures, occipital bone prominence, profound microcephaly, and normal hair pattern are consistent with the diagnosis of FBDS. His microcephaly, characterized by head circumference $Z$ score of -7.34 , was more severe than the reported average $Z$ score of -5.8 . Additionally, the neonate's brain MRI findings of occipital horn dilation and a simplified gyral pattern are consistent with colpocephaly and lissencephaly from the report of Corona-Rivera et al. Although the cause of FBDS in this neonate is unknown, one contributing factor from the gestational history is maternal tobacco smoking. Smoking is known to increase vascular resistance of the placenta, which limits blood flow to the fetus. Using the Swedish Medical Birth Registry, information on 1,362,169 infants born during 1983-1996, Källén (2000) found a highly significant association between small head circumference for gestational age and maternal smoking. CMV and Zika virus infections were ruled out by laboratory results. There is no known association between prenatal mold exposure and microcephaly. Although the Microcephaly NGS panel, which includes the NGE1 gene, and the Lissencephaly NGS panel were normal, ALG11, which is responsible for ALG11-congenital disorder of glycosylation, was not included in these panels. In retrospect, a whole-exome sequencing test would have been a more comprehensive and cost-effective genetic strategy in this circumstance. Due to the lack of prenatal care, it is uncertain whether other environmental agents or infectious etiologies contributed to FBDS in this neonate; however, genetic counseling will be offered to this family because an autosomal recessive disorder has not been ruled out.

\section{Practical Applications:}

1. Consider the diagnoses of fetal brain disruption sequence when the pattern of microcephaly presents with scalp rugae, overriding sutures, and occipital bone prominence.

2. In infants with fetal brain disruption sequence is diagnosed, brain imaging and ophthalmologic evaluations help detect structural abnormalities of the brain and eye that may require further intervention.

3. Investigate deletions on chromosome microarray, especially at 16p13.11, with comprehensive genetic testing (exome) because a deletion may unmask a deleterious variant on the other intact chromosome.

4. After prenatal infection with CMV and Zika virus have been ruled out, order trio whole-exome sequencing test, which is more comprehensive and less costly than serial gene panels that may miss some causative genes. Offer genetic counseling to families of infants with unexplained severe congenital microcephaly, because autosomal recessive traits could increase recurrence risks for future pregnancies.

5. Refer to early infant stimulation and other intervention programs for children with developmental and intellectual delays.

\section{References}

1. 1- CDC COVID-19 Response Team. Coronavirus Disease 2019 in Children - United States, February 12-April 2, 2020. MMWR Morb Mortal Wkly Rep. 2020; 69 (14):422. Epub 2020 April 10

2. Breslin, N. B.-B. (2020). COVID-19 infection among asymptomatic and symptomatic pregnant women: Two weeks of confirmed presentations to an affiliated pair of New York City hospitals. American Journal of Obstetrics \& Gynecology MFM, 100118. 
3. Sutton, D. K. (2020). Universal Screening for SARS-CoV-2 in Women Admitted for Delivery. New England Journal of Medicine.

4. Chandrasekharan, P. V.-C.-2. (2020). Neonatal Resuscitation and Postresuscitation Care of Infants Born to Mothers with Suspected or Confirmed SARS-CoV-2 Infection. American Journal of Perinatology.

5. Tran, K. C.-S. (2012). Aerosol generating procedures and risk of transmission of acute respiratory infections to healthcare workers: a systematic review. Plos one.

6. Puopolo, K. M. (2020). Management of Infants Born to Mothers with COVID-19. American Academy of Pediatrics Committee on Fetus and Newborn, Section on Neonatal-Perinatal Medicine, and Committee on Infectious Diseases.

7. Dubler, S. Z. (2016). Bacterial and viral contamination of breathing circuits after extended use - an aspect of patient safety? Anaesthesiologica Scandinavica.

8. Zeng, H. X. (2020). Antibodies in infants born to mothers with COVID-19 pneumonia. JAMA.

9. Yu, N. L. (2020). Clinical features and obstetric and neonatal outcomes of pregnant patients with COVID-19 in Wuhan, China: a retrospective, single-centre, descriptive study. The Lancet Infectious Diseases.

10. Zeng, L. X. (2020). Neonatal early-onset infection with SARS-CoV-2 in 33 neonates born to mothers with COVID-19 in Wuhan, China. JAMA pediatrics.

11. Chen H, G. J. (2020). Clinical characteristics and intrauterine vertical transmission potential of COVID-19 infection in nine pregnant women: a retrospective review of medical records. Lancet.

12. Cao, Q., Chen, Y. C., Chen, C. L., \& Chiu, C. H. (2020). SARS-CoV-2 infection in children: Transmission dynamics and clinical characteristics. J Formos Med Assoc, 119(3), 670-673.

13. Xia, W. S. (2020). Clinical and CT features in pediatric patients with COVID-19 infection: Different points from adults. Clinical Pathology.

14. Zhu, H. W. (2020). Clinical analysis of 10 neonates born to mothers with 2019-nCoV pneumonia. Translational Pediatrics, 51.

15. Wang, L., Shi, Y., Xiao, T., Fu, J., Feng, X., Mu, D., ... \& Lu, G. (2020). Chinese expert consensus on the perinatal and neonatal management for the prevention and control of the 2019 novel coronavirus infection. Annals of Translational Medicine, 8(3).

Disclosure: The authors have no disclosures

NT

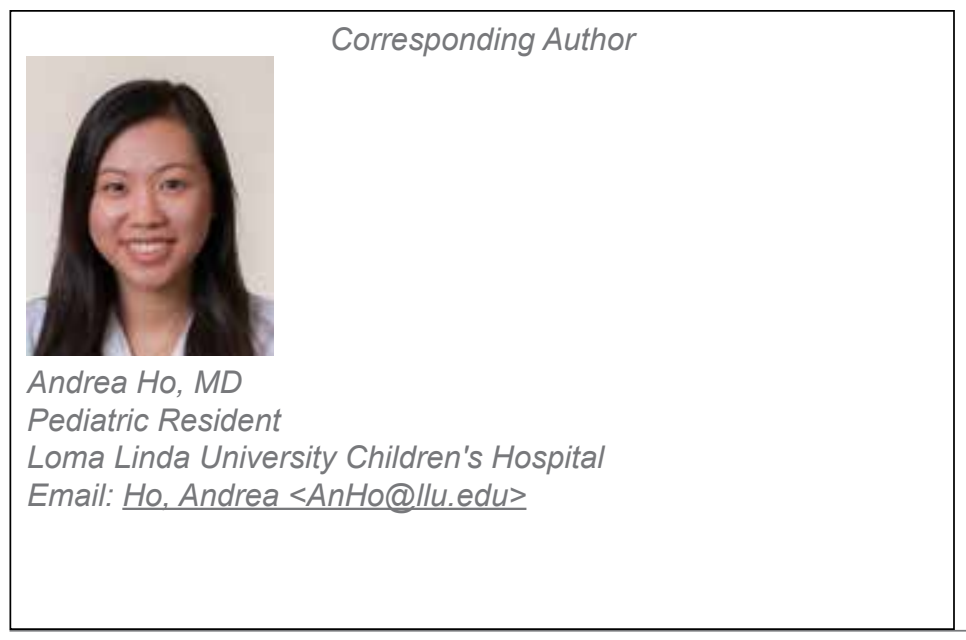

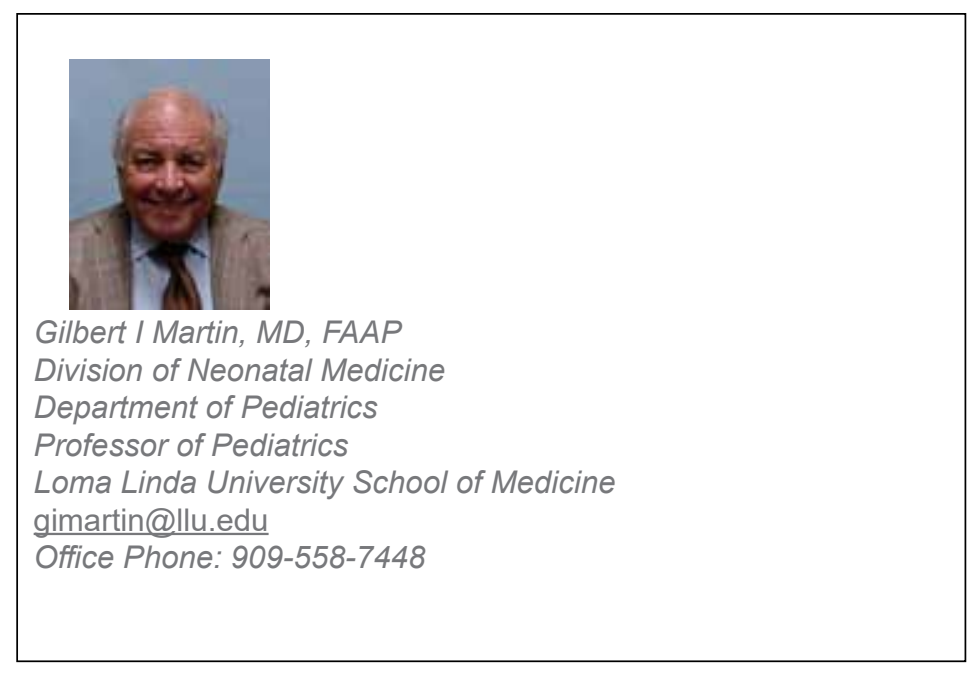

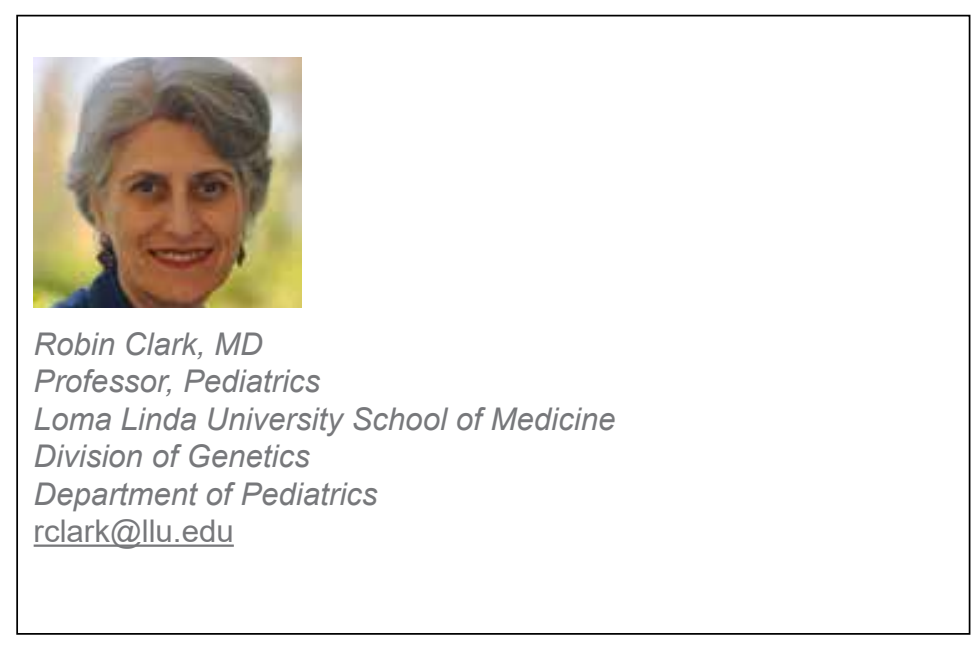

\section{Fellow's Column is published monthly.}

- Submission guidelines for "Fellow's Column":

- 2000 word limit not including references or title page. Exceptions will be made on a case by case basis

- $\quad$ QI/QA work, case studies, or a poster from a scientific meeting may be submitted..

- Submission should be from a resident, fellow, or NNP in training.

- Topics may include Perinatology, Neonatology, and Younger Pediatric patients.

- $\quad$ No more than 20 references.

- Please send your submissions to:

Elba Fayard, MD

Interim Fellowship Column Editor LomaLindaPublishingCompany@gmail.com 